\title{
A Case of Fetal Diagnosis of Noncompaction Cardiomyopathy and Coarctation of the Aorta
}

\author{
Katherine Jacobs, $\mathrm{DO}^{1} \quad$ Lauren Giacobbe, $\mathrm{MD}^{1}$ \\ Shanthi Sivanandam, MD² \\ ${ }^{1}$ Division of Maternal-Fetal Medicine, Department of Obstetrics, \\ Gynecology, and Women's Health, University of Minnesota, \\ Minneapolis, Minnesota \\ 2 Division of Pediatric Cardiology, Amplatz Children's Hospital Heart \\ Center, University of Minnesota, Minneapolis, Minnesota \\ Am J Perinatol Rep 2014;4:45-48.
}

Marijo Aguilera, $\mathrm{MD}^{1} \quad$ Kirk Ramin, $\mathrm{MD}^{1}$

\begin{abstract}
Address for correspondence Katherine M. Jacobs, DO, 606 24th Avenue South, Suite 401, Minneapolis, MN 55454 (e-mail: Gran0254@umn.edu).
\end{abstract}

\author{
Abstract \\ Keywords \\ - fetal \\ - noncompaction \\ cardiomyopathy \\ - coarctation of the \\ aorta
}

Background Left ventricular noncompaction (LVNC) cardiomyopathy is a rare form of cardiomyopathy. It is difficult to diagnose prenatally and therefore not well described in the fetal population. There have been a few reports in the literature detailing isolated cases of fetal and neonatal LVNC cardiomyopathy.

Case Report We present a case of LVNC cardiomyopathy and coarctation of the aorta detected prenatally at $29+6$ weeks of gestation with survival in infancy. This is the first case report in the literature describing the fetal diagnosis of noncompaction cardiomyopathy and associated coarctation of the aorta; a rare combination.

Conclusion With a high index of suspicion, the antenatal diagnosis of noncompaction cardiomyopathy may improve neonatal morbidity and mortality.

\section{Case Report}

A 30-year-old primigravida was referred for evaluation and transfer of care due to multiple fetal abnormalities at 29 weeks of gestation. At $22+2$ weeks of gestation she had a targeted ultrasound at an outside facility which revealed severe bilateral hydronephrosis and hydroureters as well as thickened and dilated fetal ventricular walls. A genetic amniocentesis was performed and the fetal karyotype was normal with no 22q deletion.

The patient relocated and was evaluated with a targeted ultrasound and fetal echocardiogram at our facility at $29+6$ weeks of gestation. Ultrasound showed normal fetal growth, severe bilateral hydronephrosis $(20 \mathrm{~mm})$ and hydroureters, and polyhydramnios with an amniotic fluid index of 29.2. Detailed fetal cardiac evaluation revealed an enlarged cardiac size, trabeculated left ventricular apex, moderately diminished systolic function, and dilation of her ascending aorta with hypoplasia of the transverse aortic arch. The fetal echocardiographic findings were consistent with left ventric- ular noncompaction (LVNC) cardiomyopathy with associated coarctation of the aorta (-Fig. 1 ).

The maternal echocardiogram did not show evidence of LVNC. The patient also had a fetal magnetic resonance imaging, pediatric urology consultation, and neonatal intensive care unit (NICU) consultation. She underwent a primary cesarean section at term to facilitate coordination among the multiple pediatric subspecialists. The female infant weighed $3,350 \mathrm{~g}$ with Apgar scores of 8 and 9 at 1 and 5 minutes, respectively.

The infant required intubation shortly after admission to the NICU and inotropic therapy with dobutamine, epinephrine, and milrinone. Postnatal echocardiogram confirmed noncompaction of the left ventricle and severely depressed contractility with an ejection fraction (EF) of $<15 \%$ ( - Fig. 2). There was a critical postductal coarctation of the descending thoracic aorta and a dilated aortic root $(15 \mathrm{~mm})$. On day of life (DOL) 1, the infant was taken to the operating room for repair of her coarctation. The procedure was complicated by cardiac arrest and therefore the coarctation repair was aborted. The received

November 26, 2013 accepted after revision January 7, 2014

published online

April 2, 2014
DOI http://dx.doi.org/ 10.1055/s-0034-1371750. ISSN 2157-6998.
Copyright $\odot 2014$ by Thieme Medical Publishers, Inc., 333 Seventh Avenue, New York, NY 10001, USA. Tel: +1(212) 584-4662.
License terms

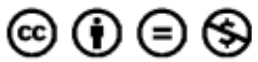




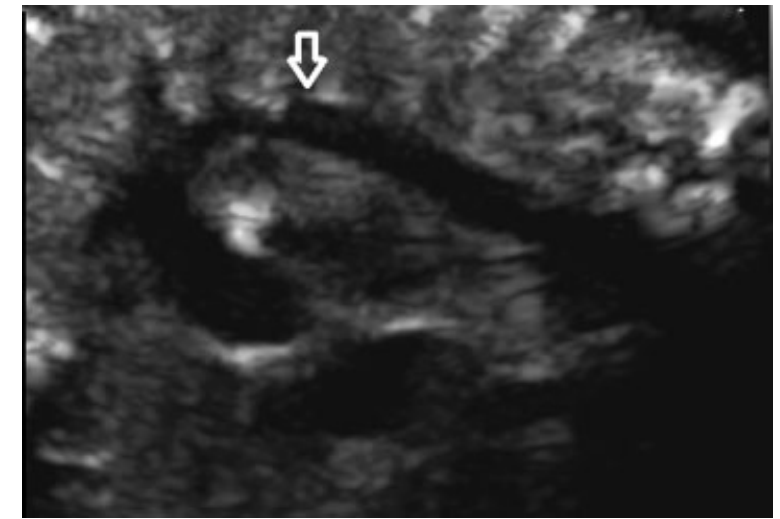

Fig. 1 Fetal echocardiogram of the aortic arch at $29+6$ weeks demonstrating postductal coarctation of the aorta. "Posterior shelf" is labeled with white arrow.

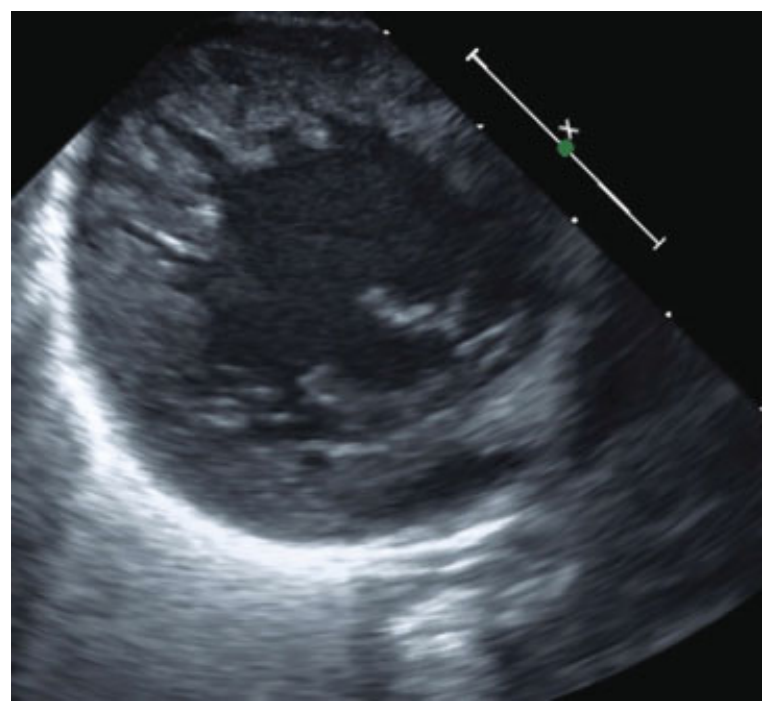

Fig. 2 Short axis postnatal echocardiogram image of the left ventricle demonstrating noncompaction of the myocardium.

following day the infant had a cardiac catheterization and stent placement to relieve the coarctation of the aorta. Despite correction of the coarctation, the infant's cardiac function did not improve. She was listed for cardiac transplantation on DOL 5.

Cord blood revealed a normal array comparative genomic hybridization. Genetic testing for cardiomyopathy, Marfan, and Barth syndrome was negative. The infant's cardiovascular status then improved in response to medications. The neonate was extubated and weaned from inotropic support. While her heart still demonstrates continued left ventricular systolic dysfunction with an EF of 36\%, she is clinically stable. She remains on aspirin, losartan, spironolactone, and furosemide. She is off the transplant list and was discharged home from the hospital at 15 weeks of age.

\section{Discussion}

Noncompaction cardiomyopathy is a rare form of cardiomyopathy that has seldom been described prenatally. It is

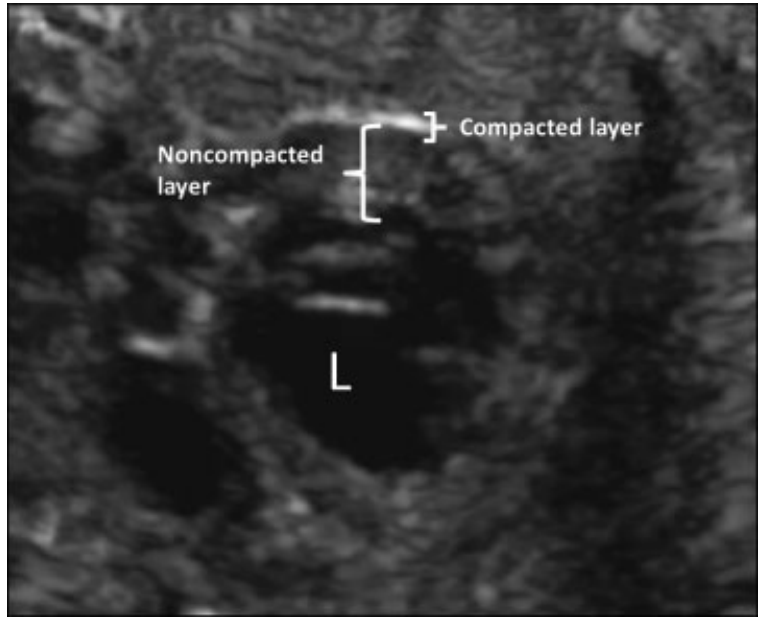

Fig. 3 Short axis fetal echocardiographic image showing the left ventricle $(\mathrm{L})$ with two distinct layers of myocardium; a thin compacted layer and a "noncompacted layer" with deep trabeculations and crevices.

characterized by deep trabeculations in the muscle of the left ventricle forming a "spongy" myocardium. This results in two layers of myocardium, an outer compacted layer and an inner noncompacted layer ( - Fig. 3 ). The spongiform layer can lead to epicardial and myocardial dysfunction resulting in poor contractility and poor filling. LVNC is a congenital abnormality that is suspected to occur due to a morphogenetic abnormality involving an arrest of compaction of the loose myocardial meshwork during fetal ontogenesis. ${ }^{1}$ This occurs between the 5th and 8th weeks of gestation. LVNC is almost invariably associated with other congenital cardiac malformations ${ }^{2}$ including atrioventricular canal defects, double-outlet right ventricle, valvular atresias, ventricular septal defect, and transposition of the great arteries. ${ }^{3}$

We present a case of the prenatal diagnosis of postductal coarctation of the aorta and LVNC, a rare combination. The ability to recognize LVNC in the fetus is difficult; therefore, it has rarely been described in this population. ${ }^{3}$ In addition, detection of coarctation of the aorta is also challenging prenatally. Coarctation of the aorta can be a progressive lesion in utero with relative worsening of the distal arch hypoplasia in later gestation, due to continual decrease in the amount of blood flow traversing the isthmus as pregnancy progresses. ${ }^{4}$ Quantitative distal aortic arch hypoplasia and ventricular and great artery size discrepancy have been observed and may facilitate the diagnosis of coarctation of the aorta during serial echocardiographic evaluation. ${ }^{5}$ Although distinct criteria have been established for the diagnosis of LVNC after birth, criteria for diagnosis in the fetus have not been established. ${ }^{3}$ Fetuses with structural cardiac defects and LVNC have a poorer prognosis than isolated LVNC. In a case series by Arunamata et al, the neonatal mortality rate including progression to heart transplantation among the fetuses with coexisting heart defects was $81 \%^{3}$ There are currently no published data on the long-term outcome of fetuses diagnosed with noncompaction and depressed ventricular function. ${ }^{6}$ The fetal presentation of LVNC can vary greatly from the 
hydropic fetus with dilated, dysfunctional, and thick-walled ventricles to the patient with complex congenital heart disease or less dramatic ventricular involvement. ${ }^{7}$

Treatment of LVNC depends on the presence or absence of other cardiac defects and the clinical manifestations of heart failure, arrhythmias, and systemic embolic events. ${ }^{6}$ Expression of disease in the fetus may be more severe than presentation later in life ${ }^{3}$ particularly if LVNC is coupled with other cardiac abnormalities. Heart transplantation is a last resort for these patients. However, there may be a role for fetal listing for neonatal heart transplantation in these rare cases of LVNC with significant ventricular dysfunction. In our case, renal anomalies were also diagnosed prenatally, which precluded fetal listing for cardiac transplantation. She was listed for cardiac transplantation on DOL 5 when relief of the coarctation and inotropic support did not appear to improve the neonate's cardiac status. Listing fetuses as candidates for neonatal heart transplantation requires careful attention to the severity of the cardiac abnormalities, certainty of an undesirable outcome without transplantation, the probability of success of transplantation, ${ }^{8}$ and consideration of extracardiac anomalies.

Technical improvements in ultrasound equipment and advances in operator training have increased the detection of fetal cardiac defects, ${ }^{6}$ allowing for a multidisciplinary team approach in planning for delivery and neonatal stabilization. LVNC and coarctation of the aorta are cardiac birth defects that can be recognized prenatally with a high index of suspicion, therefore, making the transition to postnatal life more successful. To our knowledge, this is the first reported case of prenatally diagnosed LVNC associated with coarctation of the aorta with survival in infancy.

\section{Funding}

No financial support was used for this study by any of the authors.

Conflict of Interest

None of the authors have a conflict of interest.

\section{References}

1 Aras D, Tufekcioglu O, Topaloglu S, et al. Non-compaction cardiomyopathy associated with myocardial bridging: A frequently overlooked or misdiagnosed cardiomyopathy. Eur J Heart Fail 2006; 8(7):760-763

2 Chin TK, Perloff JK, Williams RG, Jue K, Mohrmann R. Isolated noncompaction of left ventricular myocardium. A study of eight cases. Circulation 1990;82(2):507-513

3 Arunamata A, Punn R, Cuneo B, Bharati S, Silverman NH. Echocardiographic diagnosis and prognosis of fetal left ventricular noncompaction. J Am Soc Echocardiogr 2012;25(1):112-120

4 Hornberger LK, Sahn DJ, Kleinman CS, Copel J, Silverman NH. Antenatal diagnosis of coarctation of the aorta: a multicenter experience. J Am Coll Cardiol 1994;23(2):417-423

5 Gidvani M, Ramin K, Gessford E, Aguilera M, Giacobbe L, Sivanandam S. Prenatal diagnosis and outcome of fetuses with doubleinlet left ventricle. Am J Perinatal Rep 2011;1:123-128

6 Tsapakis EG, Eleftheriades M, Daskalakis G, Chrelias C, Hassiakos D. Prenatal diagnosis of fetal left ventricular non-compaction cardiomyopathy. Ultrasound Obstet Gynecol 2012;39(5):592-594

7 Menon SC, O'Leary PW, Wright GB, Rios R, MacLellan-Tobert SG, Cabalka AK. Fetal and neonatal presentation of noncompacted ventricular myocardium: expanding the clinical spectrum. J Am Soc Echocardiogr 2007;20(12):1344-1350

8 Michaels MG, Frader J, Armitage J. Ethical considerations in listing fetuses as candidates for neonatal heart transplantation. JAMA 1993;269(3):401-403 